\title{
Cumplimiento de los protocolos de trabajo seguro en alturas en la empresa MEXICHEN
}

\author{
Compliance with safe work protocols in heights at MEXICHEN
}

Zabaleta Torres Ricardo¹, Pinzón Mondol Reynaldo², Barcasnegra Madera Wendy², Morales Orozco Yefrin²

\section{Resumen}

Introducción. Ante la necesidad de mitigar los accidentes y muerte por actividades de trabajo en altura superior a 1.50 metros o más, o sobre un nivel inferior, se realiza esta investigación donde se evidencian los índices de casos presentados, condiciones de seguridad apropiadas y las recomendaciones para la utilización de equipos para la seguridad de los empleados y a su vez una formación teórico-práctica específica de los mismos. Objetivo. Valorar los lineamientos de la implementación del cumplimiento del protocolo de trabajos seguro en alturas por los trabajadores de la empresa MEXICHEN de la zona industrial de Mamonal, en Cartagena de Indias, en el año 2016. Materiales y Métodos. Observacional-descriptivo de corte transversal. El presente es un estudio que se realizó por medio de una encuesta y una lista de chequeo de equipos, la cual brindó la información necesaria para concluir con esta fase del proyecto de investigación. Resultados. El conocimiento de los contratistas existe, al aplicar la encuesta se logró apreciar que los trabajadores se desenvuelven de manera correcta en esta parte. Conclusiones. Se puede afirmar que los trabajadores de MEXICHEN Cartagena cuentan con las competencias para realizar correctamente su labor, por tal motivo están cumpliendo con el protocolo de trabajo en alturas, así como el uso de elementos para tal fin. Recomendaciones: Brindar entrenamiento teórico-práctico al personal que labora sobre 1.50 metros para que fortalezcan su conocimiento y experiencia sobre este, de esta manera mitigar las caídas con consecuencias lamentables, es importante en estas tareas mantenerse anclados a sus equipos.

Palabras claves: sistemas anti-caídas, líneas de vida, prevención de accidentes.

\footnotetext{
1. Coordinador Seguridad y Salud en el Trabajo y Medio Ambiente, Instructor PROBOARD FIRE I NFPA 1041 e Instructor NFPA 1006 de la University of Texas A\&M Engineering TEXX Extensión Service, Instructor OFDA LAC Y MSTA - USAID - SBV - SAV - TRAUMA, Especialista en Seguridad y Salud en el Trabajo, Instructor Especialista en Búsqueda - Salvamento y Rescate Avanzado, Docente del Programa de Atención Pre Hospitalaria. Facultad de Ciencias de la Salud de la Corporación Universitaria Rafael Núñez. ORCID: https://orcid.org/0000-0002-6616-9242

2. Estudiante del Programa de Atención Pre hospitalaria. Facultad de Ciencias de la Salud de la Corporación Universitaria Rafael Núñez

Bogotá, Colombia.
} 


\section{Abstract}

Introduction. In view of the need to mitigate accidents and death from work activities at heights higher than 1.50 meters or more, or at a lower level, this investigation is carried out where the rates of cases presented, appropriate safety conditions and recommendations for the use of equipment for the safety of employees and, in turn, specific theoretical and practical training of the same. Objective. To evaluate the guidelines for the implementation of compliance with the protocol of safe work at heights by the workers of the company MEXICHEN of the Mamonal - Cartagena in 2016. Materials and Methods. Observational descriptive cross-section. This is a study that was carried out through a survey and a checklist of equipment, since it is providing the necessary information to conclude this phase of the research project. Results. With this investigation it was found that the knowledge of the contractors exists, when applying the survey it was possible to appreciate that the workers are working correctly in this part. Conclusions. It can be affirmed that the workers of MEXICHEN Cartagena have the competences to carry out their work correctly, for this reason they are complying with the work protocol at heights, as well as the use of elements for that purpose. Recommendations: Provide theoretical and practical training to personnel who work on 1.50 meters to strengthen their knowledge and experience on this, in this way mitigate the falls with unfortunate consequences, it is important in these tasks to stay anchored to their equipment.

Keywords: anti fall systems, life lines, accident prevention.

\section{Introducción}

La presente investigación aborda el cumplimiento del protocolo de trabajo seguro en alturas, que establece lineamientos y procedimientos que se deben realizar durante la práctica de esta actividad, con el objetivo de prevenir cualquier accidente e incidente que acontezca durante el tiempo de ejecución. Las características principales de este tipo de trabajo es la peligrosidad a la que se ven expuestos cada día los trabajadores que la ejercen. Para analizar esta problemática es necesario mencionar, entre otras, la caída libre que es la principal causa de muerte en los últimos tiempos.

Para la realización de este trabajo se tuvieron en cuenta varios aspectos. En primer lugar, se escogió una empresa de la zona industrial de la ciudad de Cartagena de Indias; llamada MEXICHEM, que es un proveedor líder de productos y soluciones a través de múltiples sectores de petroquímica para la construcción, la infraestructura, la agricultura, la salud, el transporte, las telecomunicaciones y la energía, entre otros. MEXICHEM es uno de los mayores productores de tubos de plástico y conexiones en todo el mundo y una de las mayores empresas químicas y petroquímicas en América Latina. Actualmente tiene una planta en Cartagena, en la zona industrial de Mamonal.

Por otra parte, establecieron los indicadores socioeconómicos de los empleados que ejercen el trabajo en altura dentro de la mencionada empresa, y su nivel de escolaridad. Así 
mismo, se determinaron estadísticas recientes sobre este problema.

Para esta investigación se utilizó un instrumento (encuesta) a trabajadores que realizan trabajo en alturas y una lista de chequeo que se basó en la resolución No. 1409 de 23 de julio del 2012 del Ministerio de Trabajo (todo protocolo de trabajo en altura que se implemente debe ser guiada por esta Resolución). Para esto se utilizó un tipo de estudio Observacional - Descriptivo, con cohorte transversal. Con esta se puede analizar la situación del cumplimiento de trabajadores en una muestra determinada, este tipo de muestra fue la que empleamos en la metodología para nuestra investigación. El objetivo de indagar entre esta temática es determinar qué nivel de conocimiento tienen los trabajadores con respecto al trabajo seguro en alturas; de acuerdo con la resolución No. 1409 del 23 de julio de 2012 expedida por el Ministerio de Trabajo de Colombia y examinar la correcta utilización, manejo y mantenimiento de los elementos necesarios estipulados para realizar los trabajos seguros en alturas (3).

Los trabajos en alturas nos han permitido introducir mejoras en muchos ámbitos para tratar de eliminar, deducir y controlar riesgos. En concreto hemos experimentado un espectacular desarrollo en técnicas, metodologías y materiales específicos para la prevención de los riesgos derivados de la realización de trabajos en altura gracias, en gran medida, a la aplicación del conocimiento de la física más elemental y la concienciación preventiva (4).

Las caídas por altura se minimizaron, gracias al desarrollo de los protocolos de trabajo seguro en alturas, que mostraron técnicas es- pecíficas para el beneficio del trabajador y el empleador (1).

Así, se redujo el número de accidentes y previnieron desastres, aunque según cifra de la ARL SURA el 14\% de accidentes de trabajo mortales se dan por caídas en alturas y cifras del Instituto de Medicina Legal muestran que el $28.5 \%$ de muertes en Colombia se da por caída en alturas (2).

En esta ciudad la prensa informa con frecuencia la ocurrencia de accidentes que involucra a personas o trabajadores que se desempeñan en grandes alturas (3), por lo que se presume que no se manejan de manera adecuada los protocolos de trabajo seguro en alturas, ya sea por desconocimiento de un porcentaje de los lineamientos que la rigen o por imprudencia de quienes realizan este tipo de labor, lo cual conlleva al aumento de los índices de morbi-mortalidad en estos escenarios, creando así un problema sustancial que impacta directamente a los obreros y empleadores, ya que después de un accidente de trabajo no solo se afecta la salud de un individuo, sino también genera descompensación en todo un grupo de trabajo, afectándolos psicológicamente (1).

La investigación de esta problemática se realiza por el interés de saber qué tanto conocen los trabajadores de esta empresa el protocolo de trabajo seguro en altura y sus lineamientos que se deben implementar durante el tiempo de exposición. Esto permite identificar qué tanto ejercen las acciones que se mencionan en el protocolo, la aplicabilidad de éste dentro de la empresa y sus respectivos procedimientos y que para este artículo se toma como base los liderados por la empresa M.U. \& ASOCIADO LTDA donde se enuncia paso 
a paso como mantenerse arriba y seguro en el desarrollo de la tarea (7).

\section{Materiales y métodos}

Este estudio fue de tipo observacional - descriptivo, con corte transversal, pues describió las variables del estudio y sus magnitudes además de analizar la situación de trabajadores en una muestra determinada. Además, se buscó dar a conocer las variables de estudio del cuadro operacional mediante preguntas y respuestas en los trabajadores que realizan trabajo en altura en la empresa MEXICHEN. El estudio tuvo en cuenta 40 personas a quienes se aplicó una encuesta, y una lista de chequeo para equipos. La población que se analizó corresponde al personal que se encuentra trabajando en la empresa MEXICHEN, correspondiente a los que laboran por encima de $1,5 \mathrm{~m}$. el tipo de muestreo que se utilizó fue un muestreo intencional, no probabilístico con voluntarios.

\section{Resultados}

La encuesta aplicada a los trabajares que laboran en la empresa MEXICHEN Colombia de la ciudad de Cartagena de Indias se aplicó a trabajadores de la jornada diurna y nocturna. Se ejecutó en dos intervenciones por la variación de horario laboral (turnos de 8 y 12 horas que tiene los trabajadores). El número de encuestados fue de 40 personas que constituyen un 33\% de la población (120 en total). Se encontró que los trabajadores están en un rango de 25 a 54 años de edad. De formación de bachillerato y técnica, de sexo masculino, quienes ingresan a su lugar de trabajo con sus elementos de protección personal que les brinda la empresa.

Tabla 1. Variables sociodemográficas.

\begin{tabular}{|c|c|c|c|}
\hline MACROVARIABLE & VARIABLE & FRECUENCIA & PORCENTAJE \\
\hline \multirow{3}{*}{ EDAD } & $24-35$ & 10 & $25 \%$ \\
\hline & $36-45$ & 24 & $60 \%$ \\
\hline & $46-55$ & 6 & $15 \%$ \\
\hline \multirow{2}{*}{ DURACIÓN DEL TURNO } & 12 HORAS & 6 & $15 \%$ \\
\hline & 8 HORAS & 34 & $85 \%$ \\
\hline \multirow{3}{*}{ TIPO DE VINCULACIÓN } & EMPLEADO & 39 & $97.5 \%$ \\
\hline & CONTRATISTA & 0 & 0.00 \\
\hline & SUPERVISOR & 1 & $2.5 \%$ \\
\hline \multirow{2}{*}{ TIEMPO DE VINCULACIÓN } & AÑOS (1-5) & 16 & $40 \%$ \\
\hline & 6 O MAS AÑOS & 24 & $60 \%$ \\
\hline \multicolumn{2}{|c|}{ TOTAL } & 40 & $100 \%$ \\
\hline
\end{tabular}

Fuente. Elaboración propia basada en los datos recolectados. 
Se encontró que el $90 \%$ de los encuestados conocen la ley que regula los trabajos en altura, (Resolución No. 1409 del 23 de julio de los protocolos utilizados en las labores de trabajo seguro en alturas. Mientras que el otro 2012) y afirman saber sobre la aplicación de

Gráfico 1. Conocimiento sobre leyes regulatorias.

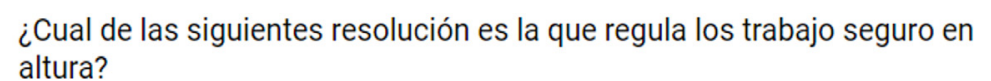
altura?

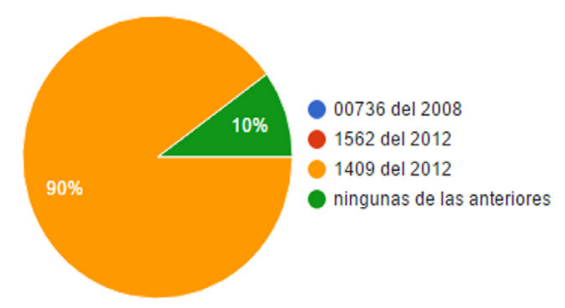

Fuente. Elaboración propia basada en los datos recolectados.

Los trabajadores de MEXICHEM dentro de boral, presencia de condición insegura o ensus estándares de seguridad industrial son torno donde los sistemas integrales de gestión muy juiciosos, se observa en la diligencia del lo exijan, de este el 100\% respondió acertadaprocedimiento de trabajo seguro y que debe mente. Ver gráfico 2. ser revisado y ajustado según la condición la-

Gráfico 2. Conocimientos sobre revisión y ajustes.

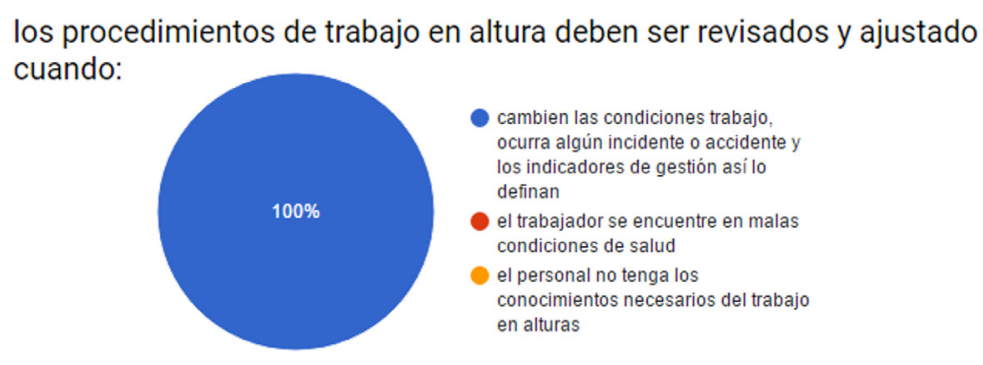

Fuente. Elaboración propia basada en los datos recolectados.

Para el tema de seguridad en los sistemas y los equipos, se observa que el total de los cuarenta (40) trabajadores encuestados tienen la precaución de revisar los equipos antes y después de utilizarlos, es una política institucional adoptada y da muestra del nivel de conciencia y autocuidado de su personal. Ver gráfico 3 . 
Gráfico 3. Revisión de equipos.

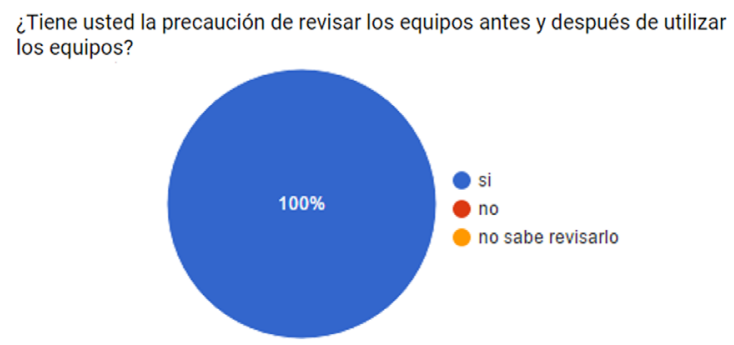

Fuente. Elaboración propia basada en los datos recolectados.

\section{Discusión}

Por todos los lineamientos de esta investigación, cuyo factor fundamental es la de reducir la accidentalidad y muerte de trabajadores por caída en alturas, se confronta los datos dados a partir del 1 de agosto de 2014, en donde las empresas de construcción, telecomunicación, mantenimiento, hidrocarburos, transporte entre otras, cuyos trabajadores desarrollan actividades, con riesgos de caída en alturas, deben estar capacitadas de acuerdo con los parámetros de ley en protección contra caídas. En Colombia según el Ministerio del Trabajo, 1283 personas murieron en los dos últimos años realizando trabajos en alturas. Gracias a actividades de prevención Positiva ARL ha disminuido los accidentes mortales en sus empresas afiliadas, en todos los sectores económicos, en el periodo enero - mayo 2014 frente a igual periodo de 2013. Las actividades laborales que requieren de trabajo en alturas (entre ellas el sector de la construcción), son las que mayor siniestralidad reportan en el país. Durante el año 2013, en Colombia, 755 personas murieron a causa de accidentes de trabajo, la mayoría de ellas, desarrollando actividades en alturas. Las cifras reportadas en Positiva revelan para ese ańo 455 accidentes fatales. De éstos, nuevamente fue el sector de la construcción el que mayor cantidad presentó con 84 muertes en el periodo analizado (5).

La empresa MEXICHEM, por estar en zona industrial maneja múltiples peligros y riesgos, según los fundamentos de trabajo en altura de nuestro País vecino Chile, el trabajo en alturas es una de las actividades laborales más peligrosas que existe; el trabajador puede verse afectado por múltiples riesgos, tales como: electrocución, quemaduras por calor, químicos y/o radiaciones (solares, UV o IR), golpes contra estructuras o por objetos en movimiento, heridas cortantes o punzantes, lesiones osteomusculares y otras que pudieran presentarse dependiendo del trabajo a realizar y las condiciones que circundan dicho trabajo, pero el riesgo especifico y principal es la caída libre (6).

En el IX Encuentro de Seguridad y Salud en el Trabajo realizado en la ciudad de Bogotá en el mes de noviembre del 2014, la directora de riesgos Laborales del Ministerio del Trabajo, Andrea Torres Matiz reportó que el sector de la construcción registra 47.579 accidentes laborales y 34 accidentes mortales con corte a junio de 2014. Entre los sectores en donde ocurren los altos índices de accidentes labora- 
les, en algunos casos por el exceso de confianza y el no uso de los implementos de trabajo, el primer lugar lo ocupa el sector inmobiliario en donde se encuentran los servicios temporales (71.919 accidentes laborales), seguido del de la industria manufacturera (50.636), y el tercer lugar se presenta en el sector de la construcción (47.579 accidentes laborales) (8).

En el recuento de los múltiples accidentes con consecuencias fatales en la ciudad de Cartagena, cabe resaltar que estos se generaron por negligencia y otras por imprudencia del trabajador, que al querer desafiar las leyes de la gravedad, no poseer el entrenamiento adecuado de trabajo en alturas y no poseer su equipo de protección contra caída EPCC; generaban accidentes con fatalidad; caso específico la caída de un trabajador (soldador) que se encontraba realizando su trabajo de soldadura en un techo en el barrio Bruselas, este señor pierde el equilibrio y cae "Soldó las bases del techo y cuando creía que había terminado se dio cuenta que le faltaba un punto de soldadura. Por eso parece que subió sin protección y cuando estaba arriba resbaló y cayó al piso, dentro del taller" (9).

Como se conoce, el nuevo Sistema de Gestión de la Seguridad y Salud en el Trabajo debe ser implementado por todas las empresas del país para proteger a todos los trabajadores, independientemente de su forma de contratación (10-14). Se pretende llegar a 550 mil empresas que implementen a cabalidad el método. La medida obliga a los contratantes a realizar un reconocimiento permanente de las condiciones de trabajo que inciden en el bienestar, la seguridad y la salud de los trabajadores, permitiendo realizar las acciones de mejora con oportunidad (15).

\section{Conclusiones}

La investigación se realizó con el fin de obtener una información acerca de cómo los trabajadores están capacitados e informados sobre los protocolos de trabajo en alturas, y como si se rigen bajo las normas que reglamentan esta actividad.

Los trabajadores de la empresa MEXICHEM de la zona industrial de Mamonal en la ciudad de Cartagena, se encuentran con las capacitaciones necesarias para laborar en trabajo seguro en alturas como lo exige la resolución.

Conflicto de Intereses: El trabajo no presenta conflicto de intereses de ninguna índole.

\section{Referencias}

1. Zabaleta T, R. Evaluación del cumplimiento de los protocolos de trabajo seguro en alturas por los contratistas que se desempeñan en el barrio Manga en la ciudad de Cartagena en el año 2013.

2. Administradora de riesgos laborales ARL - SURA. Cinco (5) éxitos en alturas. Septiembre $10 \mathrm{de}$ 2013 disponible en: https://www.arlsura.com/index.php/173-noticias-riesgos-profesionales/noticias/2047-5-exitos-en-alturas.

3. Ministerio del Trabajo. Resolución No. 1409 del 23 de Julio de 2012. Diario Oficial No. 48.517 de 9 de agosto de 2012. MINISTERIO DE TRABAJO. Por la cual se establece el Reglamento trabajo en Alturas.

4. ASPREN. Ley 31 del 1995 Madrid España. Trabajos en alturas en prevención de riesgos laborales. Disponible en: http://www.aspren.org/trabajos-altura-prevencion-riesgos-laborales/

5. Federación de aseguradores colombianos (FASECOLDA) Bogotá 24 de abril de 2014 fuente: positiva 
compañía de seguros. Disponible en: www.fasecolda. com

6. Silva V, D. manual de "seguridad para trabajo en altura" asociación chilena de seguridad. (ACHS). Chile 2010-2011. Disponible en: http://www.energygreen. cl/wp-content/uploads/2017/10/Seguridad-para-trabajos-en-altura.pdf

7. Uribe M.J. Procedimiento de trabajo en alturas M.U. \& ASOCIADO LTDA Ingeniera electromecánica y civil septiembre 27 del 2010.

8. Cabrera Q, A, Cortés V, A. Daza C, C. Caracterización de accidentalidad en una aseguradora de riesgos laborales de Colombia entre el año 2013 al 2014 disponible en: http://repository.urosario.edu.co/bitstream/handle/10336/10057/52265484-2014.pdf

9. El Universal. Muere soldador al caer de techo. publicada el 30 de marzo del 2015, disponible en: http:// www.eluniversal.com.co/sucesos/muere-soldador-alcaer-de-techo-en-el-barrio-bruselas-189127

10. Gómez, E., Rodríguez, A., Ordosgoitia, K., Rojas, M., Severiche, C. Riesgos psicosociales en personal de asistencia de una clínica de tercer nivel de la ciudad de Cartagena de Indias en 2016. NOVA. 2017; 15 (27): $77-89$

11. Ibarra, A., Rua E. Evaluación de la calidad en la atención al usuario del servicio de urgencias del hospital público de Yopal en Casanare, Colombia. NOVA. 2018; 16 (30): 21-31

12. Moreno, S., Lorena Chaparro, L., Criado, L. Vega, O., Cuenca, I. Magnitud de efecto de un programa dirigido a cuidadores familiares de personas con enfermedad crónica. NOVA. 2018; 16 (29): 11-20

13. Romero, C., Castañeda, D., Acosta, G. Determinación de la calidad bacteriológica del aire en un laboratorio de microbiología en la Universidad Distrital Francisco José de Caldas en Bogotá, Colombia. NOVA. 2016; 14 (26): 129-137

14. Sierra, D., Bedoya, E. Prevalencia de hipoacusia neurosensorial inducida por ruido en empresas del sec- tor madera de la ciudad de Cartagena. 2015. NOVA. 2016; 14 (26): 47-56

15. Ministerio del Trabajo. Decreto No. 1443 del 31 de Julio de 2014. Por la cual se establece la implementación del sistema de gestión de la seguridad y salud en el trabajo. 